\title{
Polar lattices from the point of view of nuclear spaces
}

\author{
WOJCIECH BANASZCZYK
} ABSTRACT. The aim of this survey article is to show certain questions concerning nuclear spa-
ces and linear operators in normed spaces lead to questions from geometry of numbers.

In papers [4], [5] and [6], the author considered additive subgroups of nuclear spaces. Certain results obtained there, when applied to subgroups of finite dimensional spaces and translated into the language of lattices, appear, quite unexpectedly, to be very close to the results of Hastad [11] and Lagarias, Lenstra and Schnorr [15]. These matters are discussed more thoroughly in section 1 of the present paper; it wears a survey complexion.

In sections 2 and 3 we show how the technique developed for subgroups of nuclear spaces allows to prove "ellipsoidal" analogues of the inequalities established in [15]. The results given here are new.

Motivations for studying subgroups of locally convex spaces come from functional analysis and abstract harmonic anlysis. They will be explained in monograph [7]. Whereas the investigation of connections between a lattice and its polar lattice is motivated by certain problems in geometry of numbers and, recently, in integer programming; see the introductions to [11] and [15].

The author wishes to thank H.W. Lenstra, Jr. and the referee for their remarks which contributed to an essential improvement of the paper.

\section{NOTATION AND TERMINOLOGY}

The open unit ball, the euclidean norm and the scalar product in $\mathbb{R}^{n}$ are denoted by $B_{n}, / / / /$ and (, ), respectively. The distance of a point $u$ to a set $A$ is denoted by $d(u, A)$. The linear subspace spanned over $A$ is denoted by $\operatorname{span} A$. It is convenient to set $\operatorname{span} \emptyset=\{0\}$. Thus, for a given system $u_{1}, u_{2}, \ldots, u_{m} \in \mathbb{R}^{n}$, the symbol span $\left\{u_{i}\right\}_{i<k}$ for $k=1$ should be read as $\{0\}$. Through- 
out the paper, $D$ is an open $n$-dimensional ellipsoid in $\mathbb{R}^{n}$ with centre at zero and principal semiaxes $\xi_{1} \leqslant \ldots \leqslant \xi_{\text {n. }}$

A lattice in $\mathbb{R}^{n}$ is an additive subgroup of $\mathbb{R}^{n}$ generated by $n$ linearly independent vectors. Any system of freee generators of a lattice $L$ is called a basis of $L$. The family of all latices in $\mathbb{R}^{n}$ will be denoted by $\Lambda_{n}$.

Let $L$ be a lattice in $\mathbb{R}^{*}$. The set

$$
\left\{u \in \mathbb{R}^{n}:(u, w) \in \mathbb{Z} \text { for all } w \in L\right\}
$$

is a lattice, too. We call it the polar lattice and denote by $L^{*}$. We have $\left(L^{*}\right)^{*}=L$.

For each $i=1, \ldots, n$, we define the $i$-th succesive minimum of $L$ with respect to $D$ as the infimum of all $r>0$ such that dim span $(L \cap r D) \geqslant i$; we denote this quantity by $\lambda_{i}(L, D)$. The covering radius of $L$ with respect to $D$ is the infimum of all $r>0$ such that $L+r D=\mathbb{R}^{n}$; it is denoted by $\mu(L, D)$. We shall write $\lambda_{l}(L)$ and $\mu(L)$ instead of $\lambda_{i}\left(L, B_{n}\right)$ and $\mu\left(L, B_{n}\right)$, respectively.

\section{ADDITIVE SUBGROUPS OF NUCLEAR SPACES}

It was proved in [5] that closed subgroups of nuclear spaces are weakly closed. This result is a direct consequence of the weak compactness of closed balls in Hilbert spaces and the following fact:

(1.1) Lemma. Let $K$ be an arbitrary subgroup of $\mathbb{R}^{n}$. Let $w \in \mathbb{R}^{n}$ be such that $K \cap(w+D)=\varnothing$. Suppose that $\sum_{\sum}^{n} k \xi_{k}^{-1} \leqslant 1$. Then there exists a linear functional fon $\mathbb{R}^{n}$ with $f(K) \subset \mathbb{Z}, f(w) \in[1 / 4,3 / 4]+\mathbb{Z}$ and $/ / f / / \leqslant 6$.

This is Lemma 7 of [5]. A modification of the proof allows one to replace the constant 6 by, say, $1+\frac{1}{2} e^{3 / 2}<3.241$.

Let $\{x\}$ denote the distance of a real number $x$ to the nearest integer. For each $n=1,2, \ldots$, let $a_{n}$ denote the infimum of all $r>0$ which satisfy the following condition: given $L \in \Lambda_{n}$ and $w \in \mathbb{R}^{n}$, there exists some $y \in L \Downarrow\{0\}$ such that $\|v\| \cdot d(u, L) \leqslant r\{(w, v)\}$. Putting $\xi_{1}=\ldots=\xi_{n}$ in $(1.1)$, we obtain $a_{n} \leqslant 12 n(n+1)$. A somewhat better estimate $a_{n} \leqslant 6 n^{2}+1$ was obtained by Hastad [11]. An estimate from below

$$
a_{n} \geqslant \frac{n}{2 \pi e}(1+o(I)) \quad \text { as } n \rightarrow \infty
$$

is a direct consequence of the following result: 
(1.2) Theorem (Conway, Thompson). For each $n=1,2, \ldots$, there exists an $L_{n} \in \Lambda_{n}$ with $L_{n}^{*}=L_{n}$ such that

$$
\lambda_{1}\left(L_{n}\right)^{2} \geqslant \frac{n}{2 \pi e}(I+O(I)) \quad \text { as } n \rightarrow \infty
$$

For the proof, see [16], Theorem 9.5. The actual order of magnitude of $a_{n}$ for large $n$ remains unknown.

Let $G$ be an abelian topological group. By a character of $G$ we mean a homomorphism of $G$ into the multiplicative group $\{z \in \mathrm{C}:|z|=1\}$. The set of all continuous characters of $G$ is denoted by $\hat{G}$. A subgroup $H$ of $G$ is said to be dually embeded in $G$ if each continuous character of $H$ can be extended to a continuous character of $G$.

(1.3) Proposition. Let $E$ be a topological vector space. Then the formula $\exp [2 \pi i f(u)]=\chi(u)$ defines a bijection $f \rightarrow \chi$ of the dual space $E^{\prime}$ onto $\hat{E}$.

The proof can be found e.g. in [13], (23.32)(a).

It was proved in [6] that additive subgroups of nuclear spaces are dually embedded. The proof is based on the following fact:

(1.4) Lemma. Let $K$ be a subgroup of $\mathbb{R}^{n}$ with $K \cap D=\{0\}$. For each $\chi \in \hat{K}$ one can find a linear functional $f$ on $\mathbb{R}^{n}$ such that $\exp [2 \pi i f(u)]=\chi(u)$ for all $u \in K$, and

$$
\| f / / \leqslant \frac{1}{2} e^{3 / 2}\left(\sum_{k=1}^{n} k^{2} \xi_{k}^{-2}\right)^{1 / 2}
$$

More precisely, from (1.3), (1.4) and the weak compactness of closed balls in Hilbert spaces it follows immediately that discrete subgroups of nuclear spaces are dually embedded. The extending of this result to arbitrary subgroups needs a certain additional argument.

Translated into the language of lattices, (1.4) says that

$$
\lambda_{1}(L, D) \mu\left(L^{*}, B_{n}\right) \leqslant \frac{I}{2} e^{3 / 2}\left(\sum_{k=1}^{n} k^{2} \xi_{k}^{-2}\right)^{1 / 2}
$$

for each $L \in \Lambda_{n}$; the verification of this simple fact is left to the reader.

For each $n=1,2, \ldots$, let $b_{n}$ be the infimum of all $r>0$ with the following property: for each $L \in \Lambda_{n}$ with $\lambda_{1}(L) \geqslant 1$ and each $\chi \in \hat{L}$, there exists a linear func- 
tional $f$ on $\mathbb{R}^{n}$ such that $/ / f / / \leqslant r$ and $\exp [2 \pi i f(u)]=\chi(u)$ for all $u \in L$. It is not hard to see that

$$
b_{n}=\sup \left\{\lambda_{s}(L) \mu(L *): L \in \Lambda_{n}\right\} \quad(n=I, 2, \ldots) .
$$

Putting $\xi_{1}=\ldots=\xi_{n}=1$ in (1.4), we obtain $b_{n} \leqslant \frac{3}{2} n^{3 / 2}$ for $n \geqslant 5$. From Theorem (2.14) of [15] it follows that $b_{n} \leqslant \frac{1}{2} n^{3 / 2}$ for all $n \geqslant 1$. On the other hand, (1.2) implies that

$$
b_{n} \geqslant \frac{n}{4 \pi e}(I+o(I)) \quad \text { as } n \rightarrow \infty
$$

The actual order of magnitude of $b_{n}$ for large $n$ is unknown.

(1.5) Remark. The main idea of the proofs of (1.1) and (1.4) is very similar to that of Korkine-Zolotarev bases (cf. the proof of (2.2) below). Since those bases are the main tool in [11] and [15], there is no hope to improve the estimates on $a_{n}$ and $b_{n}$ obtained in [11] and [15] by modyfying the proofs of (1.1) and (1.4). What is more, the replacement of balls by ellipsoids always leads to the worsening of the constants occuring in the estimates. The reasons are of technical nature; cf. the proof of (2.7).

(1.6) Remark. A subgroup $H$ of an abelian topological group $G$ is said to be dually closed in $G$ if, to each $g \in G \backslash H$, there corresponds some $\chi \in \hat{G}$ with $\chi(H)=\{1\}$ and $\chi(g) \neq 1$. It follows easily from (1.3) that an additive subgroup of a topological vector space is dually closed if and only if it is weakly closed. Thus we may say that closed subgroups of nuclear spaces are dually closed and dually embedded. The Pontryagin duality theorem for LCA (locally compact abelian) groups implies that closed subgroups of LCA groups are dually closed and dually embedded. These results admit the following common generalization.

Let $\mathrm{N}$ be the smallest class of abelian topological groups which contains LCA groups and nuclear spaces and is closed with respect to the operations of taking subgroups, Hausdorff quotients and arbitrary products. It turns out that closed subgroups of groups belonging to $N$ are dually closed and dually embedded. The proofs are based on (1.1) and (1.4), respectively; they will be given in [7].

(1.7) Remarks. S. Sidney [17] considered weakly dense subgroups of Banach spaces. He proved that if a Banach space $X$ has a separable infinite dimensional quotient space, then $X$ contains a weakly dense proper closed subgroup. Problem 2 of [17] is very similar to our Lemma (1.1). The answer to this problem is negative; this follows from the results of [2] and [3]. 
The positive answer to Problem 1 of [17] was obtained in [3]. It was proved there that each infinite dimensional normed space contains a discrete subgroup which is weakly dense. Next, it was proved in [2] that if a metrizable locally convex space is not nuclear, then it contains a discrete subgroup which is weakly dense in the linear subspace spanned over it. An easy argument shows that such a subgroup cannot be dually embedded.

The proofs of the results obtained in [2] and [3] are also based on certain inequalities for lattices in $\mathbb{R}^{n}$; in this case, however, of the Minkowski-Hlawka type.

\section{SUCCESIVE MINIMA}

Lagarias, Lenstra and Schnorr proved in [15] that, for each $L \in \Lambda_{n}$ with $n \geqslant 7$, one has

$$
\text { (1) } \lambda_{i}(L) \lambda_{n-i+1}\left(L^{*}\right) \leqslant \frac{1}{6} n^{2} \quad(i=1, \ldots, n)
$$

The aim of this section is to prove the following result:

(2.1) Theorem. Let $L$ be an arbitrary lattice in $\mathbb{R}^{n}$. Then

(2) $\lambda_{i}(L, D) \lambda_{n-i+1}\left(L^{*}, B_{n}\right) \leqslant e^{a / 2} \sum_{k=1}^{n} k \xi_{k}^{-1}$

for $i=1, \ldots, n$.

Putting here $\xi_{1}=\ldots=\xi_{n}=1$, we obtain inequalities (1) with a worse constant on the right side.

We shall begin with some lemmas.

(2.2) Lemma. Let $u_{1}, \ldots, u_{n}$ be a basis of a lattice $L \in \Lambda_{n}$. Let $w_{1}, \ldots, w_{n}$ be the Gram-Schmidt orthogonalization of $u_{1}, \ldots, u_{n}$. Denote

(3) $r=\left(/ / / w_{1} / /{ }^{2}+\ldots+/ / w_{n} / /{ }^{2}\right)^{1 / 2}$.

Then $\mu(L) \leqslant \frac{r}{2}$. Moreover, there is a basis $v_{1}, \ldots, v_{n}$ of $L$ such that $/ / v_{k} / / \leqslant r$ for $k=1, \ldots, n$.

Proof. We may write 


$$
\begin{aligned}
& u_{1}=w_{1} \\
& u_{2}=c_{21} w_{1}+w_{2}, \\
& \ldots \ldots \ldots \ldots \ldots \ldots \ldots \ldots \ldots \ldots \ldots \ldots \\
& u_{n}=c_{n 1} w_{1}+c_{n 2} w_{2}+\ldots+w_{n}
\end{aligned}
$$

for some coefficients $c_{i j^{\prime}}$ A simple inductive argument allows us to find integers $p_{v}$ and coefficients $c_{i j}^{\prime} \in[-1 / 2,1 / 2]$ such that

$$
\begin{aligned}
& v_{1}:=u_{1}=w_{1}, \\
& v_{2}:=p_{21} u_{1}+u_{2}=c_{21}^{\prime} w_{1}+w_{2,} \\
& \ldots \ldots \ldots \ldots \ldots \ldots \ldots \ldots \\
& v_{n}:=p_{n 1} u_{1}+p_{n 2} u_{2}+\ldots+u_{n}=c_{n 1}^{\prime} w_{1}+c_{n 2}^{\prime} w_{2}+\ldots+w_{n}
\end{aligned}
$$

Hence, by (3),

$$
/ / v_{k} / / /^{2}=/ / w_{k} / /^{2}+\sum_{i<k} / c_{k l}^{\prime} / 2 / / w_{i} / / /^{2} \leqslant \sum_{i=1}^{n} / / w_{i} / /{ }^{2}=r^{2}
$$

for $k=1, \ldots, n$. Consider the rectangular parallelepiped

$$
P=\left\{t_{1} w_{1}+\ldots+t_{n} w_{n}:-\frac{1}{2}<t_{1,+\ldots}, t_{n} \leqslant \frac{I}{2}\right\}
$$

It is clear that the family $\{P+u: u \in L\}$ is a disjoint covering of $\mathbb{R}^{n}$. From (3) we get $P \subset \frac{r}{2} \bar{B}_{n:}$ Thus $\mu(L) \leqslant \frac{r}{2}$.

(2.3) Lemma. Let $w_{1}, \ldots, w_{m}$ be the Gram-Schmidt orthogonalization of some system $u_{1}, \ldots, u_{m} \in \mathbb{R}^{n}$. If $u_{1}, \ldots, u_{m} \in D$, then $/ / w_{1} / /^{2}+\ldots+/ / w_{m} / /^{2}<\xi_{1}^{2}+\ldots+\xi_{n}^{2}$.

The proof is standard.

(2.4) Lemma. Let $L$ be a lattice in $\mathbb{R}^{n}$. Denote $s=\left(\xi_{1}^{2}+\ldots+\xi_{n}^{2}\right)^{1 / 2}$. Then there is a subset $A$ of $L \cap s B_{n}$ such that all non-zero components of the closed subgroup $L+\operatorname{span} A$ are disjoint from $D$.

Proof. If $L \cap D=\{0\}$, we may take $A=0$. In the other case, we can construct inductively a linearly independent system $u_{1}, \ldots, u_{m} \in L$ such that

(4) $u_{k} \in D+\operatorname{span}\left\{u_{l}\right\}_{i<k} \quad(k=1, \ldots, m)$

and all non-zero components of the closed subgroup $L+\mathbb{R} u_{1}+\ldots+\mathbb{R} u_{m}$ are disjoint from $D$. Denote $M=\operatorname{span}\left\{u_{k}\right\}_{k=1}^{m}$. Then $\mathbb{Z} u_{1}+\ldots+\mathbb{Z} u_{m}$ is a lattice in $M$. Let $w_{1}, \ldots, w_{m}$ be the Gram-Schmidt orthogonalization of $u_{1}, \ldots, u_{m}$. It follows from (4) that $w_{1}, \ldots, w_{m}$ is, in fact, the orthogonalization of some system of vectors belonging to $D$. Hence, by (2.3), we have $/ / w_{1} / /{ }^{2}+\ldots+/ / w_{m} / /^{2}<s^{2}$. Thus, accord- 
ing to (2.2), we can find a basis $v_{1} \ldots, v_{m}$ of the lattice $\mathbb{Z} u_{1}+\ldots+\mathbb{Z} u_{m}$ such that $\| v_{k} / /<s$ for $k=1, \ldots, m$. Now, we may take $A=\left\{v_{k}\right\}_{k=1}$.

(2.5) Lemma. If $L \in \lambda_{t}$ and $L \cap D=\{0\}$, then $\lambda_{1}\left(L^{*}\right) \leqslant n\left(\xi_{1} \ldots \xi_{n}\right)^{-1 / n}$.

This is an easy consequence of the fundamental theorem of Minkowski (see e.g. [9], Theorem 1,p. 123). It follows from (1.2) that (2.5) cannot be essentially improved.

(2.6) Proposition. If $a_{1}, a_{2}, \ldots$ is a sequence of nonnegative numbers, not all equal to zero, then

$$
\sum_{k=1}^{\infty}\left(a_{1} \ldots a_{k}\right)^{1 / k}<e \sum_{k=1}^{\infty} a_{k}
$$

For the proof, see e.g [10], 9.12.

(2.7) Lemma. Let $L$ be a lattice in $\mathbb{R}^{n}$ with $L \cap D=\{0\}$. Denote

$$
t=e^{3 / 2}\left(\sum_{k=1}^{n} k^{2} \xi_{k}^{-2}\right)^{1 / 2}
$$

Then $\mu\left(L^{*}\right) \leqslant \frac{t}{2}$. Moreover, we can find a basis $v_{1}, \ldots, v_{n}$ of $L^{*}$ with $/ / v_{k} / /<t$ for $k=1, \ldots, n$.

Proof. Let $M$ be a $k$-dimensional subspace of $\mathbb{R}^{n}$ and $\eta_{1} \leqslant \ldots \leqslant \eta_{k}$ - the principal semiaxes of the $k$-dimensional ellipsoid $D \cap M$. Then $\eta_{i} \geqslant \xi_{i}$ for $i=1, \ldots, k$. Applying this standard fact and (2.5), we shall find consecutively generators $u_{n}, u_{n-1}, \ldots, u_{1}$ of $L^{*}$ such that

$$
d\left(u_{k} \operatorname{span}\left\{u_{i}\right\}_{i<k}\right) \leqslant k\left(\xi_{1} \ldots \xi_{k}\right)^{-1 / k}
$$

for $k=I, \ldots, n$. Let $w_{1}, \ldots, w_{n}$ be the Gram-Schmidt orthogonalization of $u_{1}, \ldots, u_{n}$. Applying (2.6) and the inequality $k !>k^{k} e^{-k}$, we get

$$
\begin{gathered}
\sum_{k=1}^{n} / / w_{k} / /^{2}=\sum_{k=1}^{n} d^{2}\left(u_{k}, \operatorname{span}\left\{u_{i}\right\}_{k k}\right) \leqslant \sum_{k=1}^{n} k^{2}\left(\xi_{1} \ldots \xi_{k}\right)^{-2 / k}= \\
=\sum_{k=1}^{n} k^{2}(k)^{-2 / 4}\left(\frac{l^{2}}{\xi_{1}^{2}} \ldots \frac{k^{2}}{\xi_{k}^{2}}\right)^{1 / 1 / k}<e^{3} \sum_{k=1}^{n} k^{2} \xi_{k}^{-2}=t^{2} .
\end{gathered}
$$

The result follows now from (2.2).

Proof of (2.1). Fix an arbitrary $i=1, \ldots, n$. We have to show that (2) is satisfied. Therefore, without loss of generality, we may assume that

(5) $\lambda,(L, D)=1$.

This implies that 
(6) dim $\operatorname{span}(L \cap D)<i$.

We may assume that

$$
D=\left\{\left(x_{1}, \ldots, x_{n}\right) \in \mathbb{R}^{n}: \xi_{1}^{-2} x_{1}^{2}+\ldots+\xi_{n}^{-2} x_{n}^{2}<I\right\} .
$$

Denote $r=\sum_{n=1}^{n} k \xi_{k}^{-1}$ and $\eta_{k}=\left(r^{-1} k \xi_{j}\right)^{1 / 2}$ for $k=1, \ldots, n$. Then $\sum_{k=1}^{n} \xi_{k}^{-2} \eta_{k}^{-2}=1$. Consider the ellipsoid

$$
C=\left\{\left(x_{1}, \ldots, x_{n}\right) \in \mathbb{R}^{n} \cdot \eta_{1}^{-2} x_{1}^{2}+\ldots+\eta_{n}^{-2} x_{n}^{2}<1\right\} .
$$

It follows from (2.4) that there is a subset $A$ of $L \cap D$ such that all non-zero components of the closed subgroup $K:=L+\operatorname{span} A$ are disjoint from $C$. Denote $N=\operatorname{span} A$.

Let $N^{1}$ be the orthogonal complement of $N$ in $\mathbb{R}^{n}$ and $\pi$ the orthogonal projection onto $N^{\perp}$. Then $K \cap N^{\perp}$ is a lattice in $N^{\perp}$ with $K \cap N^{\perp} \cap \pi(C)=\{0\}$. Denote $l=\operatorname{dim} N^{\perp}$ and let $\zeta_{1} \leqslant \ldots \leqslant \zeta_{l}$ be the principal semiaxes of $C$. It is known that $\zeta_{f} \geqslant \eta_{f}$ for $j=1, \ldots, l$. By $(2.7)$, the polar lattice $\left(K \cap N^{\perp}\right)^{*}$ admits a basis $v_{1}, \ldots, v_{l}$ such that

$$
/ / v_{j} / /<e^{3 / 2}\left(\sum_{k=1}^{1} k^{2} \zeta_{k}^{-2}\right)^{1 / 2} \leqslant e^{3 / 2}\left(\sum_{k=1}^{n} k^{2} \eta_{k}^{-2}\right)^{1 / 2}=e^{3 / 2} r
$$

for $j=1, \ldots, l$. It is not difficult to see that $\left(K \cap N^{1}\right)^{*}$ is isometric to $L^{*} \cap N^{1}$. Consequently, $L^{*} \cap\left(e^{3 / 2} r B_{n}\right)$ contains at least $l$ linearly independent vectors. This means that $\lambda_{l}\left(L^{*}, B_{n}\right) \leqslant e^{3 / 2} r$. Since $A \subset L \cap D$, we have $N \subset \operatorname{span}(L \cap D)$, whence, by (6),

$$
l=\operatorname{dim} N^{2}=n-\operatorname{dim} N>n-i .
$$

Consequently, $\lambda_{n-i+1}\left(L^{*}, B_{n}\right) \leqslant \lambda\left(L^{*}, B_{n}\right) \leqslant e^{3 / 2} r$. In view of $(5)$, this proves (2).

Theorem $(2,1)$ is a new result though all tools needed in its proof can be found in [4], [5] and [6]. The proof of (2.2) follows on the lines of that of Lemma 4 in [4]. The same idea occurs in [8]; it is very similar to the idea of Korkine-Zolotarev bases (cf. (1.5)).

Lemma (2.3) is another form of Lemma 1.2 of [6]. The proof of (2.4) is essentially the same as that of Lemma 4 in [5]. Finally, the argument used in the proof of (2.7) can be found in the proof of Lemma 3 in [4]; the idea of applying (2.6) comes from [5].

\section{BIORTHOGONALITY}

The theory of nuclear groups, presented in [7] (see (1.6)), would undergo a considerable simplification if it were possible to prove the following fact: 
(3.1) Conjecture. There exist absolute constants $c>0$ and $m \in \mathbb{N}$ satisfying the following conditions: if $L \in \Lambda_{n}$ and $\xi_{k} \geqslant c k^{m}$ for $k=1, \ldots, n$, then there exists an $n$-dimensional $o$-symmetric ellipsoid $C$ in $\mathbb{R}^{n}$ with $B_{n} \subset C \subset D$, such that $L$ admits a basis which is orthogonal with respect to the scalar product defined by $C$.

Let $p$ be the Minkowski functional of $D$. It is not hard to see that (3.1) would follow from the following assertion:

(3.2) Conjecture. There exist absolute constants $c>0$ and $m \in \mathbb{N}$ satisfying the following condition; if $L \in \Lambda_{n}$ and $\xi_{k} \geqslant c k^{m}$ for $k=1, \ldots, n$, then one can find some $u_{1}, \ldots, u_{n} \in L$ and $w_{1}, \ldots, w_{n} \in L^{*}$ with $\left(u_{j}, w_{j}\right)=\delta_{j}$ for all $i, j=l, \ldots, n$, such that $/ / u_{1} / / \cdot p\left(w_{1}\right) \leq i^{-m}$ for $i=1, \ldots, n$.

It is not known nowadays whether these conjectures are true (cf. (3.5)). The aim of this section is to prove the following result, weaker than (3.2):

(3.3) Theorem. Let $p$ be the Minkowski functional of $D$. Then, for each $L \in \Lambda_{n}$, one can find some $u \in L$ and $w \in L^{*}$ with $(u, w)=l$ and

$$
\| u / / \cdot p(w) \leqslant 3\left(\sum_{k=1}^{n-1} k^{2} \xi_{k}^{-2}\right)^{1 / 2} .
$$

Proof. Without loss of generality we may assume that

$$
\begin{aligned}
& \text { (8) } \lambda_{1}\left(L, B_{n}\right)=I, \\
& \text { (9) }\left(\sum_{k=1}^{n-1} k^{2} \xi_{k}^{-2}\right)^{1 / 2}=1 / 3 .
\end{aligned}
$$

Let $e_{1}, \ldots, e_{n}$ be the unit vectors in $\mathbb{R}^{n}$. We may write $\mathbb{R}^{n-1}=\operatorname{span}\left\{e_{1}, \ldots, e_{n-1}\right\}$. By (8), there is some $u \in L$ with $/ / u / /=1$. We may assume that $u=e_{n}$. In view of (9), it is enough to prove that there is some $w \in L^{*}$ with $(u, w)=I$ and $p(w) \leqslant I$. Suppose the contrary. Then

$$
L^{*} \cap D \cap\left(e_{n}+\mathbb{R}^{n-1}\right)=\varnothing .
$$

Let $\eta_{1} \leqslant \ldots \leqslant \eta_{n-1}$ be the principal semiaxes of the $(n-l)$-dimensional ellipsoid $D \cap \mathbb{R}^{n-1}$. It is a standard fact that

$$
\xi_{k} \leqslant \eta_{k} \quad(k=1, \ldots, n-l) .
$$

Let us write $h=\sup \left\{x_{n}:\left(x_{1}, \ldots, x_{n}\right) \in D\right\}$ and let $\zeta_{1} \leqslant \ldots \leqslant \zeta_{n-1}$ be the principal semiaxes of the $(n-l)-$ dimensional ellipsoid $C=D \cap\left(e+\mathbb{R}^{n-1}\right)$. It is clear that 


$$
\zeta_{k}=\left(1-h^{-2}\right)^{1 / 2} \eta_{k} \quad(k=1, \ldots, n-l) .
$$

Let $\pi$ be the orthogonal projection onto $\mathbb{R}^{n-1}$. It is not hard to see that $K:=\pi(L)$ is a lattice in $\mathbb{R}^{n-1}$ with

$$
\lambda_{1}\left(K, B_{n-1}\right) \geqslant \frac{\sqrt{3}}{2}
$$

Next, it is clear that $L^{*} \cap \mathbb{R}^{n-1}$ is the polar lattice of $K$. Let $s$ be the centre of $C$. From (10) it follows that

$$
\left(L^{*} \cap \mathbb{R}^{n-1}\right)+(C-s) \neq \mathbb{R}^{n-1} .
$$

In view of (2.7), this implies that

$$
\lambda_{1}\left(K, B_{n-1}\right) \leqslant(1 / 2) e^{3 / 2}\left(\sum_{k=1}^{n-1} k^{2} \zeta_{k}^{-2}\right)^{1 / 2}
$$

Since $h \geqslant \xi_{1}$, from (9) we get $h \geqslant 3$. Thus, by (14), (12), (11) and (9), we have

$$
\begin{aligned}
\lambda_{1}\left(K, B_{n-1}\right) & \leqslant(1 / 2) e^{3 / 2}\left(1-h^{-2}\right)^{-1 / 2}\left(\sum_{k=1}^{n-1} k^{2} \eta_{k}^{-2}\right)^{1 / 2} \\
& \leqslant(1 / 2) e^{3 / 2} \frac{3 \sqrt{2}}{4}\left(\sum_{k=1}^{n-1} k^{2} \xi_{k}^{-2}\right)^{1 / 2}=e^{3 / 2} \frac{\sqrt{2}}{8},
\end{aligned}
$$

which contradicts (13). This completes the proof.

(3.4) Remark. For each $n=1,2, \ldots$, let $s_{n}$ be the infimum of all $s>0$ which satisfy the following condition: for each $L \in \Lambda_{n}$, one can find some $u \in L$ and $w \in L^{*}$ with $(u, w)=1$ and $/ / u / / / / w / / \leqslant s$. From (3.3) we get $s_{n} \leqslant \sqrt{3} n^{3 / 2}$. For large $n$, a much better estimate can be obtained. Let $\gamma_{n}$ 's be Hermite's constants (see [9], 38.1). Denote

$$
c=\lim \sup _{n \rightarrow \infty} n^{-1} \gamma_{n}
$$

It was pointed out to the author by H.W. Lenstra, Jr. that Theorem (2.14) of [15] implies that $s_{n}<(c / 3+O(I)) n^{3 / 2}$ as $n \rightarrow \infty$. From the result of Kabat'yanskii and Levenštein [14] it follows that $c<0.872 /(\pi e)$. Thus

$$
s_{n}<(0.034+o(1)) n^{3 / 2} \quad \text { as } n \rightarrow \infty
$$

(3.5) Remark. For each $n=1,2, \ldots$, let $z_{n}$ be the infimum of all $z>0$ with the following proper y: for each $L \in \Lambda_{n}$, one can find some $u_{1}, \ldots, u_{n} \in L$ and 
$w_{1}, \ldots, w_{n} \in L^{*}$ with $\left(u_{i}, w_{j}\right)=\delta_{i j}$ for all $i, j=1, \ldots, n$ and with $/ / u_{i} / / / / / w_{i} / / \leqslant z_{n}$ for $i=1, \ldots, n$. Babai [1], Theorem 5.1, proved that $z_{n} \leqslant(3 / \sqrt{2})^{n}$ for every $n$. Hastad [11] proved that $z_{n} \leqslant \exp \left(c n^{1 / 3}\right)$ for some constant $c>0$. It seems conceivable that $z_{n}$ might actually be bounded by a polynomial in $n$. This would be an argument supporting Conjecture (3.1).

\section{References}

[1] L. BABAI, On Lovász' lattice reduction and the nearest lattice point problem, Combinatorica 6 (1986), 1-13.

[2] M. BANASZCZYK and W. BANASZCZYK, Characterization of nuclear spaces by means of additive subgroups, Math. Z. 186 (1984), 125-133.

[3] W. BaNASZCZYK, On the existence of exotic Banach-Lie groups, Math. Ann. 264 (1983), 485-493.

[4] W. BANASZCZYK, On the existence of unitary representations of commutative nuclear Lie groups, Studia Math. 76 (1983), 175-181.

[5] W. BANASZCZYK, Closed subgroups of nuclear spaces are weakly closed, Studia Math. 80 (1984), 119-128.

[6] W. BANASZCZYK, Pontryagin duality for subgrotups and quotients of nuclear spaces, Math. Ann. 273 (1986), 653-664.

[7] W. BANASZCZYK, Additive subgroups of topological vector spaces; Theory of characters, in preparation.

[8] W. BANASZCZYK and J. GRABOWSKI, Connected subgroups of nuclear spaces, Studia Math. 78 (1984), 161-163.

[9] P.M. GRUBER and C.G. LeKKERKERKER, Geometry of Numbers, NorthHolland, Amsterdam 1987.

[10] G.H. HARDY, J.E. LiTTLEWOOD and G. POLYA, Inequalities, Cambridge University Press, Cambridge 1952.

[11] J. HASTAD, Dual Vectors and lower bounds for the nearest lattice point problem, Combinatorica 8 (1988), 75-81.

[12] J. HASTAD and J.C. LAGARIAS, Simultaneously good bases of a lattice and its reciprocal lattice, preprint.

[13] E. HEWITT and K.A. Ross, Abstract harmonic analysis, vol. I, SpringerVerlag, Berlin 1963.

[14] G.A. KABAT'YANSKIİ and V.I. LEVENŠTEIN, Bounds for packing on a sphere and in space. Problemy Peredači Informatsii 14 (1978), 3-25 (in Russian) = Problems of Information Transmission 14 (1978), 1-17.

[15] J.C. LAGARIAS, H.W. LENSTRA, Jr. and C.P. SCHNORR, Korkin-Zolotarev bases and successive minima of a lattice and its reciprocal lattice, to appear in Combinatorica.

[16] J. Milnor and D. HuSEMOLLER, Symmetric bilinear forms, SpringerVerlag, Berlin 1973. 
[17] S.J. SiDNEY, Weakly dense subgroups of Banach spaces, Indiana Univ. Math. J. 26 (1977), 981-986.

Institute of Mathematics

Lódż University

Banacha 22

90-238 Lódź, Poland 